\title{
The effect of reaming velocity on the pressure distribution in the intramedullary cavity
}

\author{
O. Gaber ${ }^{1}$, K. Behdinan ${ }^{2}$, J. de Beer ${ }^{4}$, P. Zalzal ${ }^{3}$, M. Papini ${ }^{1}$ \\ \& M. Z. Saghir ${ }^{1}$ \\ ${ }^{I}$ Department of Mechanical and Industrial Engineering, \\ Ryerson University, Canada \\ ${ }^{2}$ Department of Aerospace Engineering, Ryerson University, Canada \\ ${ }^{3}$ Mount Sinai Hospital, Canada \\ ${ }^{4}$ Henderson Hospital, Canada
}

\begin{abstract}
The effect of reaming velocity on the pressure distribution within the bone is investigated numerically by solving the full three-dimensional Navier-Stokes equations together with the continuity equation using the finite element technique. Viscosity is also varied to obtain a pressure envelop. It was found that all the experimental data follow the same trends as the envelopes predicted by the finite element model. It was clear that an increase in either the implant insertion rate or the viscosity resulted in an increase in pressure in the intramedullary canal.

Keywords: finite element, fat embolus syndrome, porous media, Darcy equation, intramedullary canal.
\end{abstract}

\section{Introduction}

Fat Embolus Syndrome (FES) is related to the extrusion of the marrow into the venous system due to the increase of intramedullary pressure from reaming the intramedullary canal, or the insertion of an intramedullary device. It develops in $0.5-2 \%$ of all patients with long bone fractures, and has been associated with high morbidity and mortality [1]. It is commonly accepted that there are three main factors contributing to the increased intramedullary pressure and embolisation of fat and marrow contents when performing orthopedic procedures 
which breech the intramedullary canal: (i) the presence of a fracture, (ii) the speed of reamer penetration, and (iii) the design of the reamer [2].

The contents of the intramedullary canal mainly consist of a highly viscous medullary fat permeated by numerous arterial and venous blood vessels. When the reamer or the hip implant is inserted, the volume of the canal decreases, and as a result, the intramedullary contents may exit the bone. While reaming the canal to prepare for device insertion, the longitudinal flutes of the reamers quickly get filled by bone debris, transforming the reamer into a hydraulic pump. This results in a high intramedullary pressure. A number of suggestions have been made in an attempt to reduce this intramedullary pressure; for example, avoiding reaming altogether, the design of reamers with deeper flutes, or the use of an irrigation suction technique. Of these suggestions, only the last has been tested and proven [3].

\section{Model description}

For simplicity, a $35 \mathrm{~cm}$ long co-axial cylinders having outer diameter of $32 \mathrm{~mm}$ is used in the numerical simulation (see Figure 1). The inner diameter is changed between 9 and $10 \mathrm{~mm}$ according to the type of reamer used. The tip of the inner cylinder surface is defined in such a way so that it moved with time in order to simulate the tip of the reamer advancing through the bone marrow cavity.

In order to accurately simulate the reaming process, a good understanding of the properties of bone and bone marrow is required. One important parameter governing the fat ejection from the bone is the bone porosity, which can vary widely depending on patient age and sex. The material properties used in the present study are summarized in Table 1. Finally, it is important to note that in the present study, it is assumed that the insertion/reaming process is isothermal, so that the energy equation is omitted from our calculation. Pressure is thus assumed to be the only driving force in the model.

Table 1: $\quad$ Physical properties of different sections of the femur.

\begin{tabular}{cccc}
\hline $\begin{array}{l}\text { Physical properties of } \\
\text { Compact Bone section }\end{array}$ & $\begin{array}{l}\text { Physical properties of } \\
\text { Medullary section }\end{array}$ \\
\hline Symbol & Value & Symbol & Value \\
\hline$\Phi$ & 0.05 & $\varphi$ & 0.95 \\
\hline$\kappa$ & $3 \times 10^{-9}-16 \times 10^{-9} \mathrm{~m}^{2}$ & $\kappa$ & $3 \times 10^{-7}-16 \times 10^{-7} \mathrm{~m}^{2}$ \\
\hline$\rho$ & $1810 \mathrm{~kg} / \mathrm{m}^{3}$ & $\rho$ & $1810 \mathrm{~kg} / \mathrm{m}^{3}$ \\
\hline
\end{tabular}




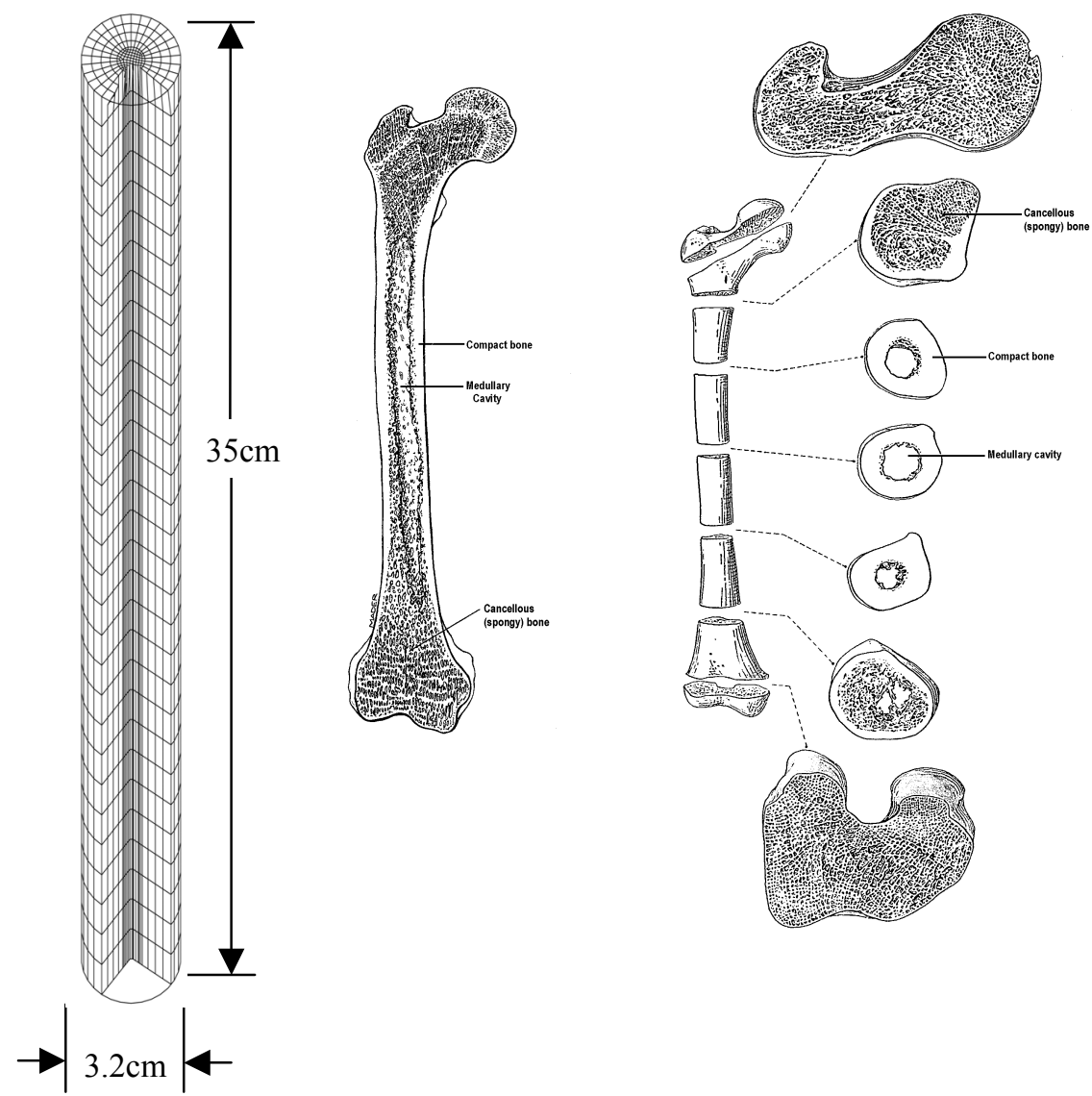

Figure 1: Numerical model of the femur as well as the femur profile [13].

\section{Governing equations}

\subsection{Navier-Stokes equations}

The three dimensional differential equations of fluid flow through a porous medium for a laminar, transient, incompressible, Newtonian flow can be written in dimensionless form as follows:

\section{R-Component}

$$
\frac{\mathrm{Re}}{\phi} \frac{\partial \mathrm{U}_{\mathrm{r}}}{\partial \tau}+\frac{1}{\mathrm{Da}} \mathrm{U}_{\mathrm{r}}=-\frac{\partial \mathrm{P}}{\partial \mathrm{R}}+\frac{1}{\mathrm{a}}\left[\frac{\partial}{\mathrm{R} \partial \mathrm{R}}\left(\frac{\partial\left(\mathrm{RU}_{\mathrm{r}}\right)}{\partial \mathrm{R}}\right)+\frac{1}{\mathrm{R}^{2}} \frac{\partial^{2} \mathrm{U}_{\mathrm{r}}}{\partial \theta^{2}}-\frac{2}{\mathrm{R}^{2}} \frac{\partial \mathrm{U}_{\theta}}{\partial \theta}+\frac{\partial^{2} \mathrm{U}_{\mathrm{r}}}{\partial \mathrm{Z}^{2}}\right]+\mathrm{Re}
$$


Ө-Component:

$$
\frac{\mathrm{Re}}{\phi} \frac{\partial \mathrm{U}_{\theta}}{\partial \tau}+\frac{1}{\mathrm{Da}} \mathrm{U}_{\theta}=\frac{1}{\mathrm{R}} \frac{\partial \mathrm{P}}{\partial \theta}+\frac{1}{\mathrm{a}}\left[\frac{\partial}{\mathrm{R} \partial \mathrm{R}}\left(\frac{\partial\left(\mathrm{RU}_{\theta}\right)}{\partial \mathrm{R}}\right)+\frac{1}{\mathrm{R}^{2}} \frac{\partial^{2} \mathrm{U}_{\theta}}{\partial \theta^{2}}+\frac{2}{\mathrm{R}^{2}} \frac{\partial \mathrm{U}_{\mathrm{r}}}{\partial \theta}+\frac{\partial^{2} \mathrm{U}_{\theta}}{\partial \mathrm{Z}^{2}}\right]
$$

\section{Z-Component}

$$
\frac{\mathrm{Re}}{\phi} \frac{\partial \mathrm{U}_{\theta}}{\partial \tau}+\frac{1}{\mathrm{Da}} \mathrm{U}_{\mathrm{Z}}=\frac{\partial \mathrm{P}}{\partial \mathrm{Z}}+\frac{1}{\mathrm{a}}\left[\frac{1}{\mathrm{R}} \frac{\partial}{\partial \mathrm{R}}\left(\frac{\mathrm{R} \partial \mathrm{U}_{\mathrm{Z}}}{\partial \mathrm{R}}\right)+\frac{1}{\mathrm{R}^{2}} \frac{\partial^{2} \mathrm{U}_{\mathrm{Z}}}{\partial \theta^{2}}+\frac{\partial^{2} \mathrm{U}_{\mathrm{Z}}}{\partial \mathrm{Z}^{2}}\right]
$$

The dimensionless variables used to obtain the above equations are as follows:

$$
\begin{gathered}
\mathrm{R}=\frac{\mathrm{r}}{\mathrm{L}}, \mathrm{Z}=\frac{\mathrm{z}}{\mathrm{L}}, \mathrm{U}_{\mathrm{r}}=\frac{\mathrm{u}_{\mathrm{r}}}{\mathrm{u}_{0}}, U_{\theta}=\frac{u_{\theta}}{u_{0}} \tau=\frac{t u_{0}}{L}, \mathrm{U}_{\mathrm{z}}=\frac{\mathrm{u}_{\mathrm{z}}}{\mathrm{u}_{0}}, \mathrm{P}=\frac{\mathrm{pL}}{\mu \mathrm{u}_{0}}, \operatorname{Re}=\frac{\rho u_{0} L}{\mu}, \\
a=\frac{\mu}{\bar{\mu}}, \mathrm{Da}=\frac{\kappa}{L^{2}} u_{0}=\sqrt{g L}, \mathrm{v}=\frac{\mu}{\rho}
\end{gathered}
$$

where $\mathrm{r}$ is the bone radius, $\mathrm{L}$ is the characteristic length of the cylindrical bone representation, $\mathrm{u}_{0}$ the characteristic velocity adopted in the non dimensionalization, $\mathrm{t}$ is the time in seconds, $\rho$ is the density in $\mathrm{Kg} / \mathrm{m}^{3}$ and $\kappa$ is the bone permeability in $\mathrm{m}^{2}$. Equations (1) to (3) describe the Momentum (Navier-Stokes) equation for the fluid flow in a porous medium, where Re is Reynolds number and Da is Darcy number. $U_{r}, U_{\theta}$ and $U_{Z}$ represent the dimensionless fluid velocity in the radial $(r)$, angular $(\theta)$ and axial $(z)$ direction, respectively. Finally, $\mu$ and $\mathrm{p}$ represent the viscosity and the pressure, respectively. The non dimensional variable a is defined as the ratio of the fluid viscosity to the effective viscosity.

\subsection{Continuity equation}

In numerical modeling, the continuity equation, Eq. (5), must also be satisfied.

$$
\frac{1}{\mathrm{R}} \frac{\partial}{\partial \mathrm{R}}\left(\mathrm{RU}_{\mathrm{r}}\right)+\frac{1}{\mathrm{R}} \frac{\partial \mathrm{U}_{\theta}}{\partial \theta}+\frac{\partial \mathrm{U}_{\mathrm{z}}}{\partial \mathrm{Z}}=0
$$

\subsection{Boundary conditions}

The predictions of the present model will be compared to the results of Johnson et al [14], who monitored the pressure distribution within a femur during intramedullary reaming. In those experiments, a frozen cadaver femur was cleaned of all flesh, and instrumented with pressure taps mounted at mid-shaft. Initially, the bone was breached with a guide wire. A $9 \mathrm{~mm}$ reamer was then used to make the initial cavity. The reaming speed was $2.58 \mathrm{~cm} / \mathrm{s}( \pm 0.6 \mathrm{~cm} / \mathrm{s})$. 
Since there was no living tissue surrounding the bone, the cavity stayed empty after the initial ream. In reality, the cavity would fill up with blood and fat instantaneously. This was simulated by refilling the bone cavity with Albumin. The second reaming was done using a $10 \mathrm{~mm}$ reamer. Pressure values were obtained for both reaming cases and were plotted. The boundary conditions used in the modeling were chosen to simulate experiments performed by Johnson et al [14] wherein Teflon was wrapped around the bone to prevent escape of intramedullary canal contents, and to maintain a constant temperature of $37^{\circ} \mathrm{C}$ (thus maintaining our assumption that the process was isothermal, and that the energy equation could be omitted for the sake of simplicity):

- Velocity was specified as zero at the surface of the outer cylinder as well as the top and bottom of the outer cylinder and the top of the inner cylinder. Therefore no fluid could escape through the side walls nor the top and bottom of both cylinders:

$$
\mathrm{U}_{\mathrm{r}}=0, \quad \mathrm{U}_{\theta}=0, \quad \mathrm{U}_{\mathrm{Z}}=0 \text { at } \mathrm{r}=1.6 \mathrm{~cm} \text { and } \mathrm{at}=0 \text { and } 35 \mathrm{~cm} \text {. }
$$

- At the tip of the inner cylinder, the surface velocity is set to be equal to the insertion rate to mimic the movement of the reamer as it advances at a constant velocity.

$$
\mathrm{U}_{\mathrm{Z}}=\text { insertion velocity }
$$

\section{Discussion}

The experiments conducted by Johnson et al [14] involved a cadaveric bone, whose exact dimensions and shape were unfortunately not reported. As a first approximation, the experiments were simulated using the concentric cylinder setup described in Figure 1. Figure 1 also shows the location of the compact bone layer as well as the intramedullary section in the femur. The inner diameter of the cylinder (mimicking the intramedullar section) was adjusted for each reamer size for a tight sealed fit. Teflon was wrapped around the bone in the experiments, so that no fluid was allowed to escape through the pores of the bone, a condition reflected in the choice of the zero velocity boundary condition on the outer surface of the cylinder.

Because the exterior surface of the femur (so called compact bone) is porous, a fluid wets the surface. Knowing the physical properties (e.g. viscosity) of this fluid is important in the determination of the pressures at different locations. This information was missing from the published paper by Johnson et al [14]. One would expect that the fluid in this region is of high viscosity which may contain mushy fluid which we was modeled as a porous medullary cavity. In the present model, the fluid in the compact bone pores was assigned a very high viscosity value in an attempt to have the inner marrow cavity pressurize in a similar way to that of the experimental value; i.e. so that the marrow was prevented from penetrating the pores of the bone so easily. The approach which is sued in our current analysis is to use the experimental results for the $9 \mathrm{~mm}$ reamer as a 
benchmark model to determine the viscosity of this fluid. The viscosity of the fluid in the bone pores was adjusted to replicate the results obtained experimentally from the $9 \mathrm{~mm}$ reamer using a marrow viscosity of $\mu=0.22 \mathrm{~Pa}$.s. These same bone viscosity values were then used to prove that the model worked well also for the $10 \mathrm{~mm}$ reamer case.

An important challenge faced by the authors was the uncertainty in, and wide range of, material and process parameters required for the analysis. The insertion rates quoted by Johnson et al [14] for their experiments varied by as much as $23 \%$, necessitating the use of a very wide parametric envelope in order to compare with the experimental data. Physical properties reported in the literature for the marrow (e.g. viscosity) [7] and bone (e.g. permeability) were also found to vary over a very large range. To establish a viable pressure envelop, the upper, lower and average values of the marrow viscosity and insertion velocities were used. Another assumption adopted in the model regards the fluid which filled the intramedullary canal (i.e. the simulated marrow). Because the fluid is mushy (i.e. fluid containing solid particle), a medium with $95 \%$ porosity was assumed to indicate that the fluid is not clear.

\subsection{Insertion with $9 \mathrm{~mm}$ reamer}

As a first test, Johnson et al inserted a $9 \mathrm{~mm}$ reamer from one side of the bone. The pressure was then monitored at mid shaft for different insertion rates.

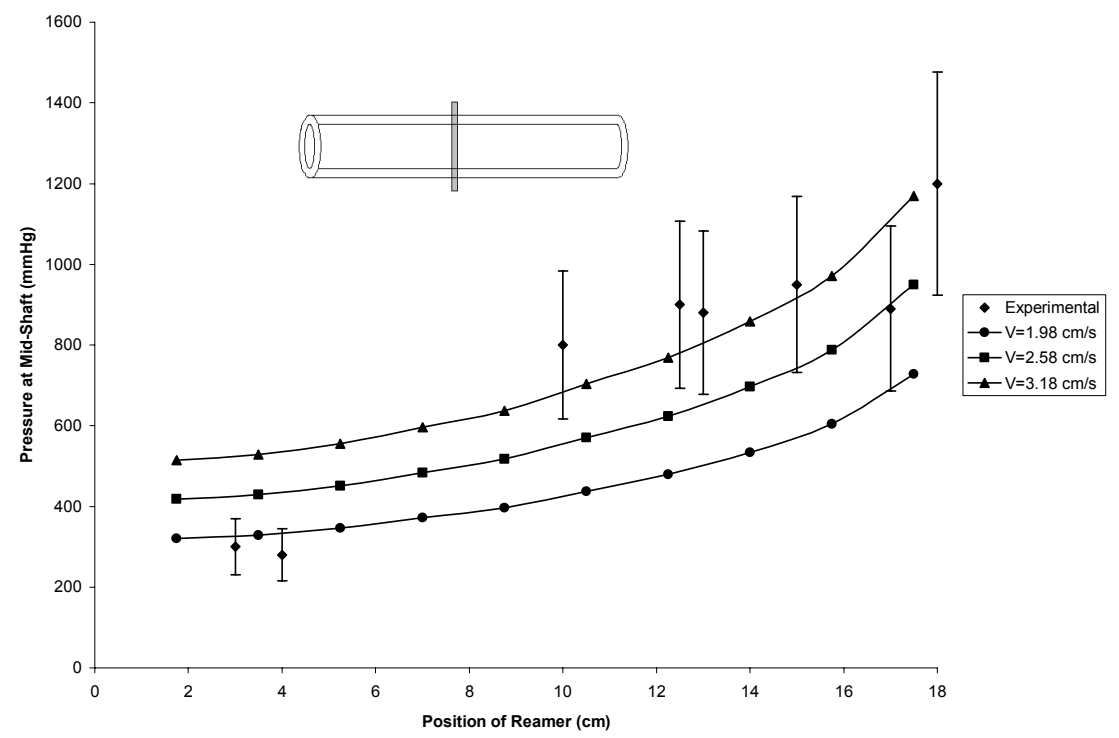

Figure 2: Pressure distribution vs. the $9 \mathrm{~mm}$ reamer position for different reamer insertion rate for an assumed viscosity of $0.22 \mathrm{~Pa}$.s.

Figure 3 presents the variation of the pressure with the reamer position for an insertion rate of $1.98 \mathrm{~cm} / \mathrm{s}, 2.58 \mathrm{~cm} / \mathrm{s}$ and $3.18 \mathrm{~cm} / \mathrm{s}$ respectively. In this figure 
the viscosity of the marrow was assumed to be equal to $\mu=0.22 \mathrm{~Pa}$.s. It is evident that, as the reamer is advanced, the pressure increases continuously in an exponential fashion. The present model assumed that backflow fluid was not allowed to escape from the interface between the bone and reamer, which is of course an idealized condition. Nevertheless, Johnson et al did not report any fluid escaping, and Figure 3 shows that the predicted trends reasonably match those shown by the experimental data.

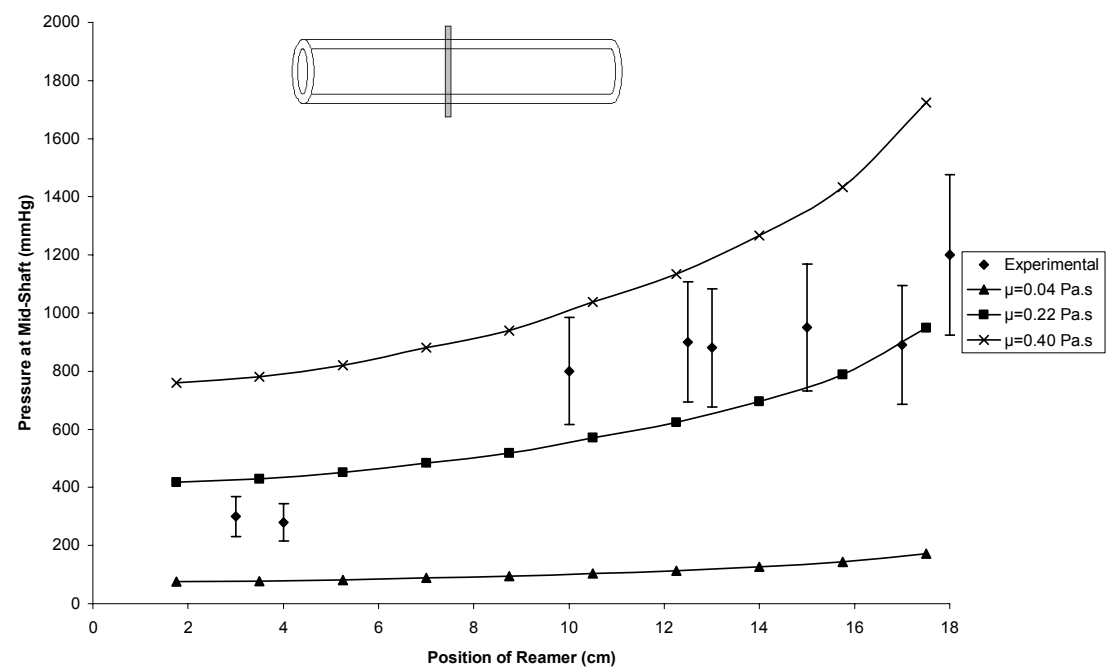

Figure 3: Pressure distribution vs. the $9 \mathrm{~mm}$ reamer position for varying marrow viscosities, for an insertion rate of $2.58 \mathrm{~cm} / \mathrm{s}$.

The effect of reamer position on intramedullary pressure is shown in Figure 4, for an insertion rate of $2.58 \mathrm{~cm} / \mathrm{s}$ and for three different marrow viscosities found in the literature. Here the pressure is monitored at the mid shaft and compared with the experimental data. The marrow viscosity clearly plays an important role in determining the pressure variation as function of the insertion rate. A viscosity of $\mu=0.22 \mathrm{~Pa}$.s results in the best agreement between numerical and experimental results.

\section{Conclusions}

It is clear that as the marrow viscosity increases, the pressure values increase. The error between the experimental and numerical results might be due to the instability of the advancement rate of the reamer in the experimental portion. The reamer advance rate varied by as much as $23 \%$ in the experimental data supplied by reference [14]. It is also observed that as the reamer insertion rate increased, 
the pressure values also increased. Overall the pressure values for both experimental and numerical tests showed consistency and followed the same trend. For the most part all pressure values fell within the envelopes calculated for the upper and lower limits of velocity and viscosity. It can thus be concluded that the use of CFD to predict the flow and pressure in the intramedullary contents of a bone is a promising tool for the study of fat embolus syndrome.

\section{Acknowledgement}

The authors would like to acknowledge the financial support of the National Science and Engineering Council (NSERC).

\section{References}

[1] Pell, Alastair, Christie, James, Keating, John, Sutherland, George. The Detection of Fat Embolism by Trasoesophageal Echocardiography Durind reamed Intramedullar Nailing. The Journal of Bone and Joint Surgery; Vol 75-B No 6, pp. 921 - 925, November 1993.

[2] Peter, R.E., Selz, T., Koestli, A. Influence of the reamer shape on intraosseus pressure during closed intramedullary nailing of the unbroken femur. Injury 1994.

[3] Sturmer K. M., Measurement of intramedullary pressure in an animal experiment and proposition to reduce the pressure increase, Injury 1993, Supplement

[4] Orr, J. F., Dunne, N. J., Development of a Computer Model to Predict Pressure Generation Around Hip Replacement Stems, Proc Instn Mech Engrs, Vol. 214 Part H (2000)

[5] Muller, Chr., McIff, T., Rahn, B.A., Pfister, U., Weller, S. Intramedullary pressure, strain on the diaphysis and increase in cortical temperature when reaming the femoral medullary cavity- a comparison of blunt and sharp reamers

[6] Smit, Theo, Huyghe, Jacques, Cowin, Stephen. Estimation $\mathrm{f}$ the poroelastic parameters of Cortical Bone. Journal of Biomechanics, 35, pp. $829-835,2002$.

[7] Bryant, J. D., David, T., Gaskell, P. H., King S. and Lond, G. Rheology of Bovine Bone Marrow. Proc. Instn Mech. Engrs 203, 71-75, 1989.

[8] Muller, Chr., Frigg, R., Pfister, U. "Effect of Flexible Drive Diameter and reamer Design on the Increase of pressure in the medullary cavity during reaming". Injury 1993, Supplement 3.

[9] Shin, Sehyun, Young-Nam, Kim, Lee, Ji-Hyung. Viscosity and Conductivity measurements of Dilute Dispersions of Rodlike Paraffin Particles in Silicone Oil. Int. comm. Heat Mass Transfer, Vol. 29 No. 2, pp. $203-211,2002$.

[10] Biyikli, S., Experimental Investigation of the Thermophysical Properties of Compact Bone, Mech. Composite Mater. Res. 20, pp. 1335 - 1345, 1985. 
[11] Grimm, Michele J. and Williams, John L. Measurements of Permeability in Human Calcaneal Trabecular Bone. Journal of Biomechanics, Vol. 30, No. 7,743-745, 1997.

[12] Cowin, Stephen. Bone Poroelasticity. Journal of Biomechanics 32, pp. $217-238,1999$.

[13] Agur, Anne, Grant's Atlas of Anatomy, $9^{\text {th }}$ edition. Williams \& Wilkins, Philadelphia, 1991.

[14] Johnson, J.A., Berkshire, A., Leighton, R. K., Gross, M., Chess, D.G., Petrie, D, Some Basic biomechanical characteristics of medullary pressure generation during reaming of the femur, Injury Vol. 26, No. 7, pp. 451-454, 1995. 